\title{
Qualidade dos sedimentos em áreas alagadas de veredas rurais e urbanas
}

As veredas, uma fitofisionomia encontrada no Cerrado responsável pela manutenção de cursos d'água de bacias hidrográficas, são consideradas áreas de preservação permanente. Portanto, seus limites, vegetações naturais e qualidade dos recursos devem ser preservados. 0 objetivo deste trabalho foi avaliar a qualidade dos sedimentos em cinco áreas alagadas de veredas na região de Uberlândia (MG), sendo três rurais e duas urbanas, por meio da análise de dezoito elementos metálicos. Os elementos avaliados foram importantes para a análise de qualidade, pois através dos métodos estatísticos utilizados foi possível diferenciar as áreas alagadas com base na composição química dos sedimentos. As alterações no uso e ocupação do solo decorrentes de atividades antrópicas, foram um dos principais fatores na diferenciação das características dos sedimentos dentre as veredas monitoradas. A área com maior quantidade de elementos em não conformidade com as legislações vigentes foi uma das veredas urbanas. O cádmio, cromo, cobre e zinco foram os metais encontrados em concentrações acima dos valores de referência de qualidade estabelecidos em Minas Gerais. Esse estudo também mostrou que as veredas com mais intervenções, exercem maior pressão na qualidade dos sedimentos do que nos ambientes preservados.

Palavras-chave: Intervenções antrópicas; Metais pesados; Quimiometria.

\section{Sediment quality in rural and urban wetlands}

Veredas, a phytophysiognomy found in the Cerrado that is responsible for maintaining watercourses in watersheds, are considered permanent preservation areas. Therefore, their limits, natural vegetation and quality of resources must be preserved. The objective of this work was to evaluate the quality of sediments in five flooded areas of veredas in the region of Uberlândia (MG), three rural and two urban, through the analysis of eighteen metallic elements. The evaluated elements were important for the quality analysis, because through the statistical methods used it was possible to differentiate the flooded areas based on the chemical composition of the sediments. Changes in land use and occupation resulting from anthropic activities were one of the main factors in the differentiation of sediment characteristics among monitored paths. The area with the largest number of elements that did not comply with current legislation was one of the urban paths. Cadmium, chromium, copper and zinc were the metals found in concentrations above the quality reference values established in Minas Gerais. This study also showed that paths with more interventions exert greater pressure on sediment quality than in preserved environments.

Keywords: Anthropic interventions; Heavy metals; Chemometrics.

Topic: Desenvolvimento, Sustentabilidade e Meio Ambiente

Reviewed anonymously in the process of blind peer
Received: 22/06/2019

Approved: 23/07/2019
Rudmir Rogério de Camargo Faxina (iD

Universidade Federal de Uberlândia, Brasil http://lattes.cnpq.br/0200245267791270 http://orcid.org/0000-0001-7376-1722 rudcmg@gmail.com

Ednaldo Carvalho Guimarães

Universidade Federal de Uberlândia, Brasil http://lattes.cnpq.br/9047680281290501 http://orcid.org/0000-0001-8328-9687 ecg@ufu.br

Sueli Moura Bertolino

Universidade Federal de Pelotas, Brasil http://lattes.cnpq.br/1154202559580478

http://orcid.org/0000-0001-5111-070X suelibertolino@ufu.br
Referencing this:

FAXINA, R. R. C.; GUIMARÃES, E. C.; BERTOLINO, S. M.. Qualidade dos sedimentos em áreas alagadas de veredas rurais e urbanas. Revista Ibero-Americana de Ciências Ambientais, v.10, n.4, p.261-272, 2019. DOI: http://doi.org/10.6008/CBPC2179-6858.2019.004.0020 


\section{INTRODUÇÃO}

Os ecossistemas de vereda são uma das principais fitofisionomias encontradas no Bioma Cerrado, sendo talvez uma das mais importantes e ao mesmo tempo sensíveis a intervenções. Existem poucos estudos sobre essas áreas, alguns relatam a biodiversidade da fauna e flora, outros as principais características dos solos. Também existem diferentes termos para sua caracterização, sendo que neste estudo adotou-se como referência a definição de Ribeiro et al. (2008), de que as veredas formam sistemas de drenagem superficial com afloramento do lençol freático dando origem a áreas alagadas em solos predominantemente hidromórficos, possuindo uma vegetação herbáceo-graminosa e outra arbóreo-arbustiva com a ocorrência de buritis na faixa central.

Apesar desta importância, as veredas têm sido progressivamente pressionadas, devido às intervenções antrópicas agrícolas e pastoris. Essas áreas estão sendo descaracterizadas pela construção de pequenas barragens e açudes, estradas, agricultura, pecuária e até mesmo por queimadas excessivas. 0 pisoteio do gado pode causar processos erosivos e compactar o solo (GUIMARÃES, 2001), afetando a taxa de infiltração de água que abastece os aquíferos subterrâneos (MEIRELLES et al., 2004). Quando em desequilíbrio, as veredas podem perder características importantes. Caso o lençol freático sofra rebaixamento devido à impermeabilização do solo, a perenidade das águas pode ser comprometida. Buritis isolados sem a ocorrência de solo encharcado podem ser um registro de uma área que anteriormente era de vereda, mas que foi alterada por intervenções antrópicas.

Por se tratarem de áreas com importância ecológica e hidrológica e serem sensíveis a alterações, as veredas são classificadas como Áreas de Preservação Permanente (APP), protegidas por Lei (MINAS GERAIS, 2013). Em ecossistemas como as veredas, o solo é tão importante quanto a fauna e flora. Em Minas Gerais, a qualidade do solo é definida pela Deliberação Normativa COPAM n.166, de 29 de junho de 2011 que lista os Valores de Referência de Qualidade (VRQ) e Valores de Prevenção (VP) para alguns elementos químicos e metálicos (MINAS GERAIS, 2011).

Nos solos podem ser encontrados diversos elementos metálicos em diferentes concentrações, sendo de origem natural ou antrópica. Entretanto, devido à alta biodisponibilidade, mobilidade e potencial toxicidade de alguns metais, é imprescindível o monitoramento destes elementos nos demais compartimentos ambientais, como nos sedimentos. O acúmulo de metais nestes ambientes, fora dos valores de referência preconizados por órgãos ambientais, se deve a intervenções antropogênicas. Alguns insumos agrícolas, por exemplo, podem liberar esses elementos no solo, ressaltando que o Brasil é um dos maiores consumidores de agroquímicos (ARAÚJO et al., 2017).

Embora esses insumos contribuem com o aumento da produção de alimentos, também liberam metais, principalmente presentes em fertilizantes $(\mathrm{Cd}, \mathrm{Cr}, \mathrm{Pb}, \mathrm{Zn}$ ) e pesticidas ( $\mathrm{Cu}, \mathrm{Pb}, \mathrm{Mn}, \mathrm{Zn}$ ) (MENDES et al., 2006; SILVA et al., 2016). Fertilizantes fabricados a partir de rochas fosfatadas podem elevar os níveis de metais como $\mathrm{Cd}, \mathrm{Cu}, \mathrm{Mn}, \mathrm{Ni}, \mathrm{Pb}$ e $\mathrm{Zn}$ no solo (FREITAS et al., 2009). A criação intensiva de animais, principalmente bovinos, suínos e aves também pode liberar $\mathrm{Cu}$, As e $\mathrm{Zn}$, por meio dos dejetos, muitas vezes 
sem o tratamento adequado (COSTA et al., 2007; FARRÉ et al., 2012).

A avaliação dos dados coletados nos sedimentos pode envolver uma grande quantidade de variáveis, cuja interpretação pode se tornar difícil, sendo necessário o uso de métodos de redução de dimensionalidade como os empregados na quimiometria, facilitando o entendimento, reduzindo a complexidade e o tempo necessário (FÁVERO et al., 2017). Dentro da quimiometria existem diferentes técnicas para análise do comportamento dos dados. Duas das mais utilizadas são as técnicas multivariadas de Análise por Componentes principais (PCA) e Análise por Agrupamento Hierárquico (HCA).

A PCA é um método utilizado para projetar dados em um subespaço menor que o inicial, facilitando a visualização das informações mais importantes e permitindo estimar a influência de cada variável em cada amostra. A HCA possui o objetivo de exibir os dados em uma única dimensão, a distância ou similaridade, de maneira a dar ênfase nos agrupamentos e padrões naturais (OTTO, 2016).

O crescimento populacional, a exploração econômica irracional dos recursos naturais e a demanda crescente por espaços estão relacionados de alguma forma com a degradação da qualidade e poluição do meio ambiente. Esses vetores de pressão são assuntos de interesse público, pois seus impactos afetam a todos de modo geral. Nesse contexto, o presente trabalho objetivou avaliar os sedimentos em função de suas características físico-químicas em cinco áreas alagadas (zona de fundo) de veredas urbanas e rurais na bacia do Rio Uberabinha, com a análise de dezoito elementos metálicos. Este estudo também pode subsidiar dados ambientais relevantes para os agricultores que usufruem das veredas para subsistência, não podendo olvidar, ainda, se tratar de um importante instrumento para a gestão pública.

\section{MATERIAIS E MÉTODOS}

\section{Área de estudo}

As áreas de estudo deste trabalho (figura 1) estão inseridas na bacia do rio Uberabinha. Essa região hidrográfica possui uma área de aproximadamente $2.200 \mathrm{~km}^{2}$ e está localizada na mesorregião do Triângulo Mineiro, abrangendo os municípios de Tupaciguara, Uberlândia e Uberaba. O clima predominante na região do município de Uberlândia é do tipo Cwa, conforme a classificação de Köppen-Geiger (ALVARES et al., 2013). A concentração de chuvas ocorre nos meses de dezembro e janeiro, com período de escassez entre maio e outubro e temperatura média anual de $23^{\circ} \mathrm{C}$ (FLAUZINO, 2014). A geologia da região é composta principalmente pelo Grupo Bauru (Cretáceo), sendo que esse conjunto sedimentar está assentado sobre rochas Pré-Cambrianas do Grupo Araxá (BACCARO et al., 2001).

O relevo é suavemente ondulado sobre formações sedimentares e a área da bacia está inserida em um conjunto de formas denominadas de Domínio dos Chapadões Tropicais do Brasil Central e Planaltos e Chapadas da Bacia Sedimentar do Paraná e o solo predominante é da ordem dos Latossolos (GROSSI, 1991; REATTO et al., 2008). Conforme o Inventário Florestal de Minas Gerais a vegetação natural predominante é composta por fitofisionomias do Cerrado (CARVALHO et al., 2008).

Dentre as veredas selecionadas para amostragem, três estão localizadas em zona rural e foram 
denominadas de vereda rural 1 (Vr1) centralizada na latitude $19^{\circ} 11^{\prime} 35.04 " \mathrm{~S}$ e longitude $48^{\circ} 11^{\prime} 51.22^{\prime \prime} \mathrm{O}$, vereda rural 2 (Vr2) latitude $18^{\circ} 59^{\prime} 10.96 " S$ e longitude $48^{\circ} 20^{\prime} 38.36^{\prime \prime O}$ e vereda rural 3 (Vr3) latitude $18^{\circ} 59^{\prime} 0.73^{\prime \prime} \mathrm{S}$ e longitude $48^{\circ} 17^{\prime} 44.01^{\prime \prime O}$. As demais áreas dentro do perímetro urbano foram nomeadas como vereda urbana 1 (Vu1), centralizada na latitude $18^{\circ} 57^{\prime} 11.70^{\prime \prime}$ S e longitude $48^{\circ} 16^{\prime} 23.88^{\prime \prime}$ O e vereda urbana 2 (Vu2) latitude $18^{\circ} 58^{\prime} 6.55^{\prime \prime} \mathrm{S}$ e longitude $48^{\circ} 16^{\prime} 0.61^{\prime \prime} \mathrm{O}$.

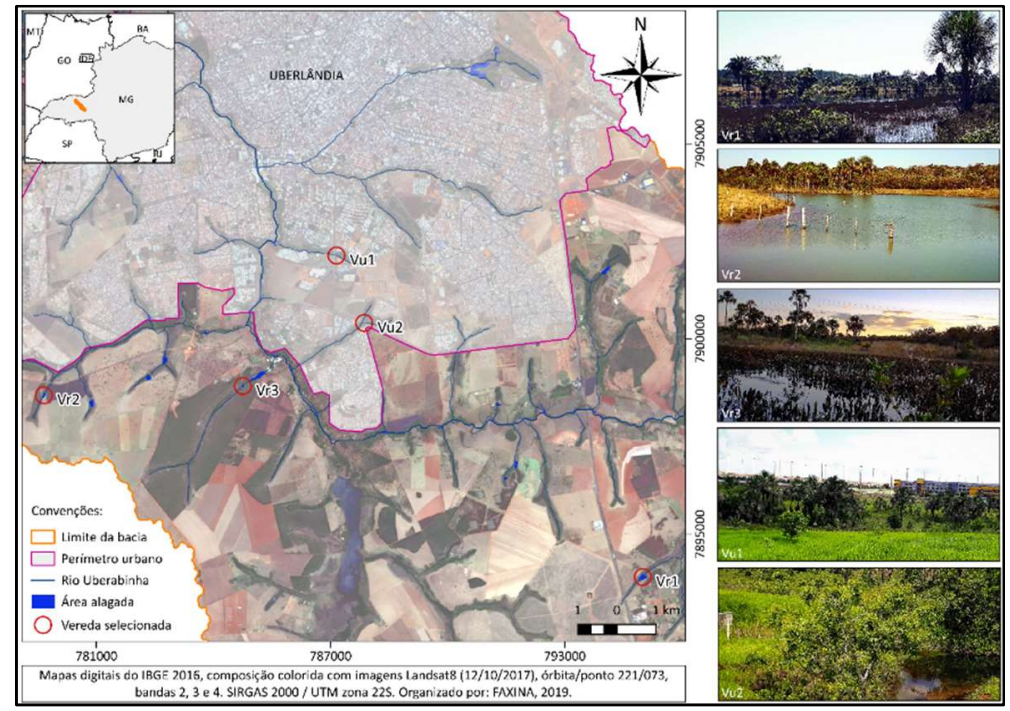

Figura 1: Localização das veredas selecionadas na bacia do Rio Uberabinha.

Plano de amostragem e análises físico-químicas

A campanha de amostragem foi realizada em agosto de 2018, com a coleta de 34 amostras simples de $0,5 \mathrm{~kg}$ de sedimentos, sendo 5 em cada vereda urbana e 8 nas rurais, em pontos distribuídos conforme a dimensão de cada área alagada. As amostragens foram realizadas com um dispositivo pressurizado confeccionado em PVC, na profundidade de 0 a $0,05 \mathrm{~m}$. As amostras coletadas foram secas ao ar, destorroadas, homogeneizadas e passadas em peneira ABNT 50, obtendo-se terra fina seca ao ar (TFSA).

Para avaliação da qualidade dos sedimentos, foram realizadas análises físico-químicas dos elementos metálicos $\mathrm{Al}, \mathrm{Ba}, \mathrm{Be}, \mathrm{Ca}, \mathrm{Cd}, \mathrm{Cr}, \mathrm{Cu}, \mathrm{Fe}, \mathrm{K}, \mathrm{Li}, \mathrm{Mg}, \mathrm{Mn}, \mathrm{Mo}, \mathrm{Na}, \mathrm{Ni}, \mathrm{Pb}, \mathrm{V}$ e $\mathrm{Zn}$, por espectrometria de emissão óptica com plasma indutivamente acoplado (ICP-OES) marca Agilent Technologies modelo 5100. Os elementos metálicos foram selecionados conforme as orientações da Deliberação Normativa COPAM no 166 de 2011 (MINAS GERAIS, 2011).

Os teores totais dos elementos metálicos nos sedimentos foram extraídos pelo método conhecido como água régia, uma modificação do método EPA 3051A (MCGRATH et al., 1985; USEPA, 1998). Triplicatas de subamostras de $500 \mathrm{mg}$, foram adicionadas em tubos de borosilicato e misturadas com $9 \mathrm{~mL}$ de $\mathrm{HCL}$ (32\%) e $4 \mathrm{~mL}$ de $\mathrm{HNO}_{3}$ (65\%) (3:1) concentrados de pureza analítica, deixando em repouso para pré-digestão por 12 horas em bloco digestor.

Posteriormente, foram submetidas ao aquecimento em três estágios: a $105^{\circ} \mathrm{C}$ por uma hora; elevação da temperatura a $140^{\circ} \mathrm{C}$ até ponto de secura; e o resíduo foi ressuspendido com $12 \mathrm{~mL}$ de $\mathrm{HCL}$ ( $20 \%$ $\mathrm{v} / \mathrm{v}$ ) e novamente aquecido em bloco digestor por mais 20 minutos a $80^{\circ} \mathrm{C}$. A solução final obtida foi 
submetida a filtragem lenta, transferida para balões e seu volume aferido para $50 \mathrm{~mL}$ com água deionizada. Para armazenamento, a solução foi transferida para tubos falcon de $50 \mathrm{~mL}$ e acondicionados sob refrigeração em geladeira até o momento da leitura.

O controle de qualidade do método utilizado para análise dos teores totais de metais nas amostras de sedimentos foi avaliado por meio das taxas de recuperação de uma amostra de referência certificada $\mathrm{BCR}^{\circ}$ - 414 (European Commission - Join Research Centre). As condições de operação do aparelho ICP-OES para as análises dos elementos metálicos nos sedimentos foram: potência RF 1200W, plasma 12 $\mathrm{Lmin}^{-1}$, auxiliar $1 \mathrm{~L}$ $\min ^{-1}$, vazão do nebulizador $0,7 \mathrm{~L} \mathrm{~min}^{-1}$, velocidade da bomba $12 \mathrm{rpm}$, tempo de estabilização $15 \mathrm{~s}$, tempo de leitura 5s. As análises foram conduzidas nos Laboratórios de Qualidade Ambiental (LAQUA/ICP) e de Pedologia (LAPED), ambos da Universidade Federal de Uberlândia (UFU).

\section{Análises estatísticas e quimiometria}

Os dados das análises dos sedimentos foram submetidos à estatística descritiva. As médias foram testadas conforme as condições de normalidade, aditividade, independência e homogeneidade para aplicação de análise de variância (ANOVA). Os elementos analisados nas amostras de sedimentos com comportamento heterogêneo e/ou sem distribuição normal foram submetidos à transformação dos dados, a saber: $B a=\sqrt{x} ; C a=\frac{1}{\log (x)} ; C u=\log (x) ; K=\sqrt{x} ; M g=\log (x) ; M n=\frac{1}{\log (x)} ; N a=\frac{1}{\sqrt{x}} ; P b=\sqrt{x}$ e as médias foram comparadas pelo teste de Tuckey.

As médias dos elementos metálicos das amostras de sedimentos foram dispostos em matriz e os dados foram submetidos a um estudo de quimiometria, com aplicação das técnicas de PCA e HCA, a fim de identificar possíveis associações entre os grupos de elementos e entre as áreas. As análises estatísticas descritiva e univariada foram executadas com o suplemento ActionStat para Excel (EQUIPE ESTATCAMP). Os métodos multivariados foram aplicados no STATISTICA (STATSOFT). Os tratamentos com análise de significância foram considerados significativos para $p<0,05$. Com os resultados das análises, foram elaborados tabelas e gráficos para classificar a qualidade dos sedimentos, identificar inconformidades com a legislação vigente e possíveis influências antrópicas nas áreas alagadas monitoradas.

\section{RESULTADOS E DISCUSSÃO}

\section{Caracterização dos sedimentos}

Os valores de recuperação dos metais para solubilização total das amostras do material de referência certificado ficaram compreendidos entre 80 a 138\%. As variações observadas estão dentro da faixa recomendada para a maioria dos elementos estudados (USEPA, 1996). Portanto, os resultados de recuperação dos analitos na amostra certificada atestam a qualidade das análises conduzidas.

Os teores totais extraídos para os elementos metálicos (tabela 1) nas amostras de sedimentos das áreas alagadas das veredas podem ser comparados com os valores encontrados nas camadas superficiais de solo de outros estudos, como os desenvolvidos por Marques et al. (2004), Ramos et al. (2006), Campos et al. 
(2013), Rosolen et al. (2015) e Ribeiro et al. (2019).

Tabela 1: Teores totais e diferenciação estatística dos elementos metálicos nos sedimentos por área monitorada.

\begin{tabular}{|c|c|c|c|c|c|c|c|c|c|c|c|c|c|c|c|c|c|c|c|c|}
\hline & \multicolumn{4}{|c|}{ Vr1 } & \multicolumn{4}{|c|}{ Vr2 } & \multicolumn{4}{|c|}{ Vr3 } & \multicolumn{4}{|c|}{ Vu1 } & \multicolumn{4}{|c|}{ Vu2 } \\
\hline \multicolumn{21}{|c|}{$\left(\mathrm{g} \mathrm{Kg}^{-1}\right)$} \\
\hline $\mathrm{Al}$ & 39,69 & \pm & 11,06 & $a$ & 28,21 & \pm & 7,72 & $a b$ & 24,16 & \pm & 10,05 & $\mathrm{~b}$ & 21,25 & \pm & 10,83 & $\mathrm{~b}$ & 24,16 & \pm & 10,50 & $b$ \\
\hline $\mathrm{Ca}^{*}$ & 0,27 & \pm & 0,09 & $a$ & 0,27 & \pm & 0,11 & $a$ & 0,22 & \pm & 0,08 & $a$ & 4,44 & \pm & 2,73 & $b$ & 0,20 & \pm & 0,10 & $a$ \\
\hline $\mathrm{Fe}$ & 15,54 & \pm & 4,59 & $a b$ & 10,84 & \pm & 3,07 & $b$ & 10,73 & \pm & 7,22 & $\mathrm{~b}$ & 19,59 & \pm & 3,00 & $b$ & 7,20 & \pm & 4,84 & $b$ \\
\hline $\mathrm{K}^{*}$ & 0,15 & \pm & 0,05 & bc & 0,10 & \pm & 0,03 & $b$ & 0,12 & \pm & 0,05 & $b$ & 0,44 & \pm & 0,20 & $a$ & 0,10 & \pm & 0,04 & $b$ \\
\hline Mg* & 0,10 & \pm & 0,01 & bc & 0,11 & \pm & 0,05 & $b$ & 0,14 & \pm & 0,06 & $b$ & 0,87 & \pm & 0,48 & $a$ & 0,12 & \pm & 0,05 & $b$ \\
\hline $\mathrm{Na}^{*}$ & 0,21 & \pm & 0,03 & $a$ & 0,19 & \pm & 0,01 & $a$ & 0,20 & \pm & 0,03 & $a$ & 0,83 & \pm & 0,43 & $b$ & 0,19 & \pm & 0,02 & $a$ \\
\hline \multicolumn{21}{|c|}{$\left(\mathrm{mg} \mathrm{Kg}^{-1}\right)$} \\
\hline $\mathrm{Ba}^{*}$ & 13,13 & \pm & 4,94 & bc & 4,64 & \pm & 1,39 & $d$ & 15,94 & \pm & 5,21 & $b$ & 70,32 & \pm & 21,89 & $a$ & 7,01 & \pm & 3,31 & $\mathrm{~cd}$ \\
\hline $\mathrm{Be}$ & 0,10 & \pm & 0,04 & $a b$ & 0,06 & \pm & 0,01 & $b$ & 0,15 & \pm & 0,05 & $a$ & 0,17 & \pm & 0,08 & $a$ & 0,10 & \pm & 0,08 & $a b$ \\
\hline $\mathrm{Cd}$ & 3,33 & \pm & 1,40 & $a b$ & 2,12 & \pm & 0,73 & $b$ & 2,28 & \pm & 1,90 & $\mathrm{~b}$ & 4,94 & \pm & 1,27 & $a$ & 1,57 & \pm & 0,72 & $\mathrm{~b}$ \\
\hline $\mathrm{Cr}$ & 56,66 & \pm & 9,06 & $a$ & 52,94 & \pm & 7,83 & $a$ & 83,28 & \pm & 33,63 & $a$ & 78,89 & \pm & 14,11 & $a$ & 74,69 & \pm & 47,03 & $a$ \\
\hline $\mathrm{Cu}^{*}$ & 20,59 & \pm & 5,57 & bc & 14,86 & \pm & 2,65 & $b$ & 16,14 & \pm & 6,93 & $b$ & 59,37 & \pm & 26,56 & $a$ & 36,01 & \pm & 39,43 & $a b$ \\
\hline $\mathrm{Li}$ & 2,09 & \pm & 0,59 & bc & 1,63 & \pm & 0,45 & $b$ & 1,70 & \pm & 0,81 & $a b$ & 3,46 & \pm & 1,13 & $a$ & 2,86 & \pm & 2,07 & $a b$ \\
\hline $\mathrm{Mn*}$ & 60,52 & \pm & 19,95 & $a$ & 69,23 & \pm & 12,17 & $a$ & 105,65 & \pm & 149,90 & $a$ & 234,12 & \pm & 102,40 & $b$ & 57,79 & \pm & 14,05 & $a$ \\
\hline Mo & 0,54 & \pm & 0,16 & $a b$ & 0,72 & \pm & 0,09 & $a$ & 0,54 & \pm & 0,12 & $a b$ & 0,43 & \pm & 0,22 & $b$ & 0,49 & \pm & 0,07 & $a b$ \\
\hline $\mathrm{Ni}$ & 11,71 & \pm & 2,08 & bc & 10,67 & \pm & 1,23 & $b$ & 12,95 & \pm & 3,36 & $a b$ & 15,63 & \pm & 2,27 & $\mathrm{a}$ & 11,47 & \pm & 2,21 & $\mathrm{~b}$ \\
\hline $\mathrm{Pb}^{*}$ & 5,89 & \pm & 2,54 & $a b$ & 2,84 & \pm & 0,70 & $b$ & 5,91 & \pm & 2,82 & $a b$ & 10,73 & \pm & 2,77 & $a$ & 6,82 & \pm & 6,12 & $a b$ \\
\hline $\mathrm{V}$ & 82,00 & \pm & 19,10 & $a b$ & 68,83 & \pm & 15,54 & $a b$ & 84,00 & \pm & 35,79 & $a b$ & 102,56 & \pm & 8,19 & $a$ & 55,55 & \pm & 15,14 & $b$ \\
\hline $\mathrm{Zn}$ & 36,24 & \pm & 12,81 & $b$ & 75,56 & \pm & 29,52 & $b$ & 275,60 & \pm & 64,23 & $a$ & 76,02 & \pm & 40,96 & $b$ & 84,46 & \pm & 29,24 & $b$ \\
\hline
\end{tabular}

Legenda: * Elemento submetido à transformação dos dados. Médias seguidas por letras iguais horizontalmente, não diferem entre si pelo teste de Tuckey $(\alpha=0,05)$.

As diferenças entre os teores naturais de metais em solos podem ser atribuídas, principalmente ao material de origem e a fatores pedogenéticos (GUILHERME et al., 2005). Em relação aos sedimentos, essa afirmação corrobora parcialmente com os baixos teores encontrados nas veredas monitoradas, visto que toda a região do Triângulo Mineiro incluindo a bacia do Rio Uberabinha foi esculturada em rochas sedimentares, sobretudo do Grupo Bauru, representadas pelos arenitos das Formações Marília, Adamantina e Uberaba, e da Formação Botucatu do Grupo São Bento e encontra-se assentada sobre rochas PréCambrianas, representadas principalmente pelos xistos do Grupo Araxá (BACCARO, 1991).

Solos derivados de rochas sedimentares e ígneas ácidas cristalinas apresentam teores mais baixos de metais quando comparados aos solos originados de rochas básicas, especialmente as máficas que são mais ricas em metais (PAYE et al., 2010). Todas as áreas monitoradas estão localizadas sobre regiões com a predominância da mesma classe de solo, Latossolo Vermelho-Amarelo Distrófico, conforme o Mapa Temático de Geodiversidade de Minas Gerais organizado pelo Serviço Geológico do Brasil (CPRM, 2010). Ramos et al. (2014), em um estudo de classificação do solo de três diferentes veredas na região do Triângulo Mineiro, encontrou solos hidromórficos pertencentes às classes dos Organossolos, Gleissolos Melânicos e Gleissolos Háplicos associados à variação do lençol freático.

A influência da pedogênese não é óbvia, pois os teores médios de metais podem variar amplamente entre amostras diferentes de solos pertencentes a uma mesma classe (PAYE et al., 2010). Essas variações nos teores médios também foram observadas em solos por outros autores, como Fadigas et al. (2006) e Paye et al. (2010), os quais justificam essas diferenças em função das variações nas suas propriedades físico-químicas. Para os sedimentos, outro fator que pode exercer influência nas variações dos elementos metálicos são as atividades antrópicas. As alterações nos usos e ocupações do solo podem contribuir com a adição de metais não naturais em uma determinada área. Variações dos teores superiores aos valores de referência 
estabelecidos nas legislações vigentes podem ser consideradas como contaminação.

A Deliberação Normativa do COPAM n.166 de 2011 lista os valores orientadores de alguns elementos metálicos para fins de referência de qualidade (VRQ) e prevenção (VP) de contaminação do solo. Dentre as substâncias listadas pelo COPAM e as analisadas nos sedimentos neste trabalho estão nove elementos (tabela 2), sendo que ainda não existem valores definidos para o $\mathrm{Al}, \mathrm{Be}, \mathrm{Ca}, \mathrm{Fe}, \mathrm{K}, \mathrm{Li}, \mathrm{Mg}, \mathrm{Mn}$ e $\mathrm{Na}$ (MINAS GERAIS, 2011).

Tabela 2: Concentrações médias $\left(\mathrm{mg} \mathrm{Kg}^{-1}\right), \mathrm{VRQ}$ e VP dos elementos metálicos nos sedimentos por área monitorada.

\begin{tabular}{|c|c|c|c|c|c|c|c|}
\hline Elemento & Vr1 & Vr2 & Vr3 & Vu1 & Vu2 & VRQ & VP \\
\hline $\mathrm{Ba}$ & 13,13 & 4,64 & 15,94 & 70,32 & 7,01 & 93 & 150 \\
\hline $\mathrm{Cd}$ & $3,33 *$ & $2,12^{*}$ & $2,28^{*}$ & $4,94^{*}$ & $1,57^{*}$ & $<0,4$ & 1,3 \\
\hline $\mathrm{Cr}$ & 56,66 & 52,94 & $83,28^{*}$ & $78,89 *$ & 74,69 & 75 & 75 \\
\hline $\mathrm{Cu}$ & 20,59 & 14,86 & 16,14 & $59,37^{*}$ & 36,01 & 49 & 60 \\
\hline Mo & 0,54 & 0,72 & 0,54 & 0,43 & 0,49 & $<0,9$ & 30 \\
\hline $\mathrm{Ni}$ & 11,71 & 10,67 & 12,95 & 15,63 & 11,47 & 21,5 & 30 \\
\hline $\mathrm{Pb}$ & 5,89 & 2,84 & 5,91 & 10,73 & 6,82 & 19,5 & 72 \\
\hline $\mathrm{V}$ & 82,00 & 68,83 & 84,00 & 102,56 & 55,55 & 129 & - \\
\hline $\mathrm{Zn}$ & 36,24 & $75,56^{*}$ & $275,60 *$ & $76,02^{*}$ & $84,46^{*}$ & 46,5 & 300 \\
\hline
\end{tabular}

Legenda: * Valor médio considerado acima do VRQ estabelecido pelo COPAM.

Em relação aos VRQ e VP estabelecidos na Resolução COPAM n.166/2011, foram observados valores médios superiores para quatro elementos metálicos $(\mathrm{Cd}, \mathrm{Cr}, \mathrm{Cu}$ e $\mathrm{Zn}$ ) nas áreas alagadas monitoradas. Sendo que a Vu1 foi o local de amostragem com maior número de inconformidades. Os elementos em maior concentração nos sedimentos analisados foram o alumínio (Al) e o ferro ( $\mathrm{Fe}$ ), ambos abundantes no grupo dos Latossolos. As concentrações de ferro (Fe) em solos de veredas são importantes por causa das reações de oxirredução que controlam a disponibilidade e mobilidade de outros elementos (RIBEIRO et al., 2019).

Para o bário (Ba) foi estabelecido pelo COPAM o valor de $93 \mathrm{mg} \mathrm{Kg}^{-1}$ como referência de qualidade do solo e $150 \mathrm{mg} \mathrm{Kg}^{-1}$ como limiar para prevenção (MINAS GERAIS, 2011). Nos sedimentos analisados, as concentrações ficaram abaixo dos valores de referência, sendo que a maior média encontrada para esse elemento foi de $70,32 \mathrm{mg} \mathrm{Kg}^{-1}$ na Vu1 e a menor 4,64 $\mathrm{mg} \mathrm{Kg}^{-1}$ na Vr2.

Em relação ao cádmio (Cd) são estabelecidos os valores $<0,4 \mathrm{mg} \mathrm{Kg}^{-1}$ como VRQ e 1,3mg Kg${ }^{-1}$ para VP (MINAS GERAIS, 2011). Entretanto, nas análises conduzidas, foram encontrados valores superiores em todas as áreas monitoradas, com destaque para a Vu1 onde a média foi de $4,94 \mathrm{mg} \mathrm{Kg}^{-1}$. Em estudo dos solos de diferentes veredas no Triângulo Mineiro, Ribeiro et al. (2019) encontrou um valor máximo de Cd inferior $\left(0,077 \mathrm{mg} \mathrm{Kg}^{-1}\right)$, assim como o valor médio $\left(1,88 \mathrm{mg} \mathrm{Kg}^{-1}\right)$ observado por Campos et al. (2013) anteriormente na mesma região. Em um estudo realizado com mais de 300 áreas alagadas abrangendo quatro diferentes Estados Norte Americanos, Jacob et al. (2013) encontrou valor médio de 0,382 $\mathrm{mg} \mathrm{Kg}^{-1}$ para esse elemento. O Cd é bioacumulativo, ou seja, também pode ter sido depositado nos sedimentos das veredas através de usos e ocupações do solo em épocas anteriores.

Os VRQ e VP definidos para o cromo (Cr) foram de $75 \mathrm{mg} \mathrm{Kg}^{-1}$ (MINAS GERAIS, 2011). Este parâmetro foi o único que não apresentou significância estatística dentre os resultados analisados. Entretanto, duas das cinco áreas (Vr3 e Vu1) apresentaram valores médios superiores a referência estabelecida pelo COPAM. 0 valor de referência de qualidade indicado para o cobre $(\mathrm{Cu})$ foi de $49 \mathrm{mg} \mathrm{Kg}^{-1}$ e o limiar de prevenção $60 \mathrm{mg}$ 
$\mathrm{Kg}^{-1}$ (MINAS GERAIS, 2011). No geral, a maioria das áreas apresentaram concentrações médias de Cu nos sedimentos inferiores ao estabelecido, sendo que na Vu1 o valor médio foi de $59,37 \mathrm{mg} \mathrm{Kg}^{-1}$.

Ribeiro et al. (2019) também encontrou valores de cobre abaixo da referência de qualidade. Enquanto Rosolen et al. (2015) observou concentrações de $\mathrm{Cu}$, variando de $31 \mathrm{mg} \mathrm{Kg}^{-1}$ a $88 \mathrm{mg} \mathrm{Kg}^{-1} \mathrm{em}$ solos na mesma região. Rosolen et al. (2015) também enfatizou que o principal risco de contaminação das veredas do Triângulo Mineiro pode advir do cromo, arsênio e cobre oriundos de insumos agroquímicos. Em relação ao molibdênio (Mo), o valor de $30 \mathrm{mg} \mathrm{Kg}^{-1}$ foi definido como limitar de prevenção e valores $<0,9 \mathrm{mg} \mathrm{Kg}^{-1}$ indicados como referência de qualidade (MINAS GERAIS, 2011). Para este elemento, todas as áreas apresentaram concentrações médias inferiores ao estabelecido.

Para o níquel (Ni) foram definidos os valores de $21,5 \mathrm{mg} \mathrm{Kg}^{-1}$ como sendo referência de qualidade e $30 \mathrm{mg} \mathrm{Kg}^{-1}$ como limiar para prevenção (MINAS GERAIS, 2011). Todas as áreas monitoradas apresentaram valores inferiores ao estabelecido como referência. Ribeiro et al. (2019) observou que o Ni não é diretamente influenciado pelas condições alternantes de oxirredução, típicas de ambientes alagados e também encontraram valores abaixo da referência na maioria das veredas estudadas.

O VRQ determinado para o chumbo (Pb) foi de $19,5 \mathrm{mg} \mathrm{Kg}^{-1}$, enquanto que o VP foi $72 \mathrm{mg} \mathrm{Kg}^{-1}$ (MINAS GERAIS, 2011). Dentre as médias dos sedimentos analisados nas áreas alagadas, não foram encontradas inconformidades. Ribeiro et al. (2019) também encontrou valores inferiores ao limiar de prevenção nos solos de veredas na mesma região. Em outros solos do mesmo bioma de Cerrado, Rosolen et al. (2015) observou variações nas concentrações de 12,1 a $81 \mathrm{mg} \mathrm{Kg}^{-1}$ de $\mathrm{Pb}$.

Para o elemento vanádio (V), a referência de qualidade é de $129 \mathrm{mg} \mathrm{Kg}^{-1}$ (MINAS GERAIS, 2011). A maior média observada foi na Vu1 $\left(102,56 \mathrm{mg} \mathrm{Kg}^{-1}\right)$ e nenhuma área apresentou valor superior ao estabelecido. O vanádio é um dos elementos mais comuns da crosta terrestre. A queima de combustíveis fósseis também libera esse elemento, sendo uma das suas principais fontes antrópicas. O nível de vanádio no solo está relacionado ao tipo de rocha geradora e pode substituir o ferro ou sorvido pelo óxido de ferro, explicando sua presença nos sedimentos (SHUQAIR, 2002).

No caso do zinco $(\mathrm{Zn})$, a referência definida foi de $46,5 \mathrm{mg} \mathrm{Kg}^{-1}$ para qualidade e $300 \mathrm{mg} \mathrm{Kg}^{-1}$ como prevenção (MINAS GERAIS, 2011). Foram observados valores acima do estabelecido como referência de qualidade em quatro das cinco áreas monitoradas e a única que apresentou valor inferior foi a Vr1 $(36,24 \mathrm{mg}$ $\left.\mathrm{Kg}^{-1}\right)$. Em estudo realizado por Marques et al. (2004), os valores de $\mathrm{Zn}$ encontrados nos solos de veredas da mesma região variaram de $5 \mathrm{mg} \mathrm{Kg}^{-1}$ a $36 \mathrm{mg} \mathrm{Kg}^{-1}$.

De acordo com a análise por componentes principais, os parâmetros originais se associaram em quatro PC que juntas explicam $86,21 \%$, identificando a importância das variações na composição química dos sedimentos. Os modelos de agrupamento hierárquico foram construídos utilizando os dados dos scores da análise por componentes principais, com o método de Ward como ligação e a distância Euclidiana, de modo a conferir maior importância aos dados que efetivamente causam variação na composição química dos sedimentos.

Em um dendograma, a similaridade é o inverso da distância no eixo $Y$, ou seja, quanto menor a 
distância entre os pontos, mais similar é o comportamento estatístico dos elementos agrupados. A distância vertical onde foi feita a ligação também possibilita inferir sobre a relação entre as variações desses elementos.

As PC 1 e PC 2 (Figura 2a) foram responsáveis por 65,96\% das variações e representatividade dos dados. A HCA reuniu os parâmetros mais semelhantes em cinco grupos (Figura 2b) cuja similaridade de cada agrupamento foi determinada através do critério da equação que segue, com $Y=50$. Tem-se: $\left(\frac{\text { Dlink }}{\text { Dmax }}\right) 100<$ Y, onde: Dlink é a distância de similaridade; Dmax a máxima distância euclidiana entre os dados e $\mathrm{Y}$ a linha de corte no dendograma.

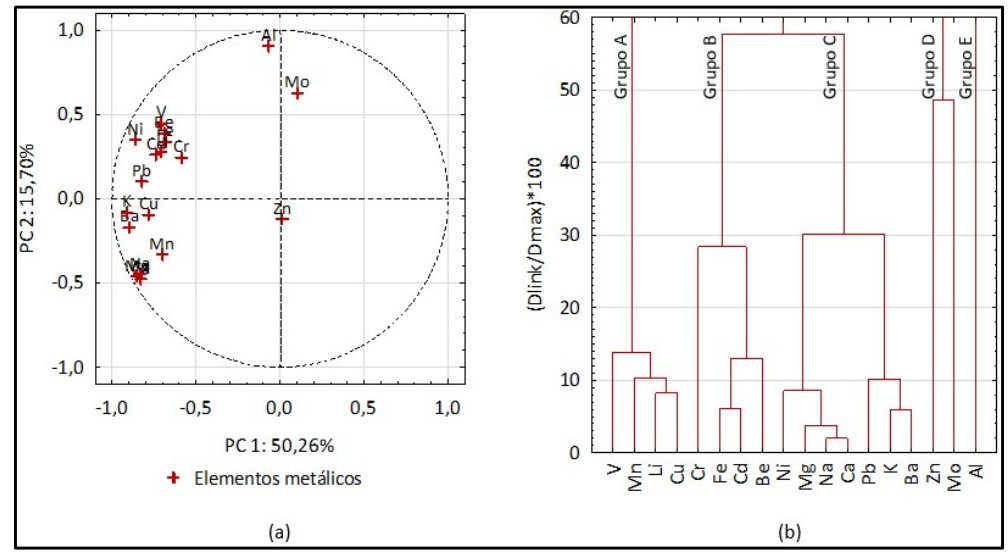

Figura 1: Associação dos elementos metálicos analisados nos sedimentos das veredas.

Este resultado mostra as variâncias dos elementos metálicos na composição química dos sedimentos, seus respectivos pesos dentro do método e associações estatísticas, como a que pode ser observada entre as bases $\mathrm{Ca}, \mathrm{Mg}$ e $\mathrm{Na}$ que também são considerados elementos litológicos. O Grupo $\mathrm{E}$, formado exclusivamente pelo Al, reflete a abundância desse elemento no Grupo dos Latossolos, característico da pedologia local.

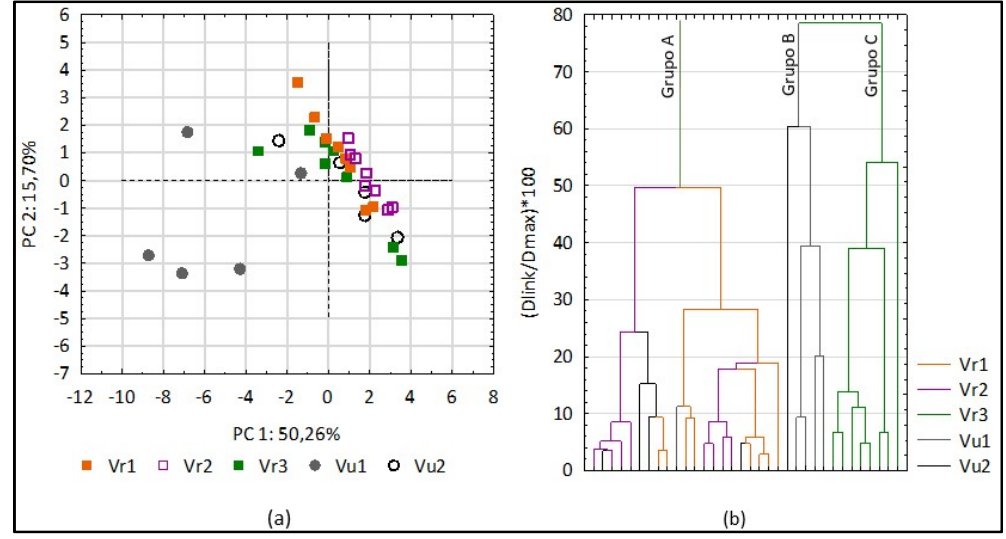

Figura 2: Classificação das veredas em função dos elementos metálicos nos sedimentos.

Os grupos $\mathrm{B}(\mathrm{Be}, \mathrm{Cd}, \mathrm{Cr}$ e $\mathrm{Fe}$ ) e $\mathrm{C}(\mathrm{Ba}, \mathrm{Ca}, \mathrm{K}, \mathrm{Mg}, \mathrm{Na}, \mathrm{Ni}$ e $\mathrm{Pb}$ ), são formados por uma mescla de indicadores geoquímicos e não geoquímicos. O Grupo $\mathrm{A}(\mathrm{V}, \mathrm{Mn}$, $\mathrm{Li}$ e $\mathrm{Cu})$ com exceção do Li, está mais relacionado com a abundância de Fe, visto que são elementos fortemente associados aos óxidos de ferro (hematita, goetita) abundantes nos Latossolos de Minas Gerais (CAIRES, 2009). O Grupo D é formado pelos elementos $\mathrm{Zn}$ e Mo. Apesar desses elementos serem mais escassos nos solos, podem ser encontrados 
facilmente em fertilizantes minerais primários.

Em relação a classificação das áreas alagadas a partir dos elementos metálicos presentes nos sedimentos, a PCA (figura 3a) revelou uma não associação estatística da Vu1 em relação às demais áreas monitoradas e uma maior homogeneidade dentre os pontos amostrados na Vr2. Na análise de HCA (figura 3b) a similaridade de cada agrupamento foi determinada através do critério da equação proposta, com $Y=$ 70 .

Observa-se uma divisão em três grupos e novamente a pouca similaridade da Vu1 em relação às demais áreas. As amostras da Vu2 apresentaram composição similar às áreas rurais Vr1 e Vr2. Essa similaridade pode ser justificada primeiramente por ambas estarem localizadas sobre o mesmo tipo de solo, e também pela condição atual de uso e ocupação do solo na Vu2 visto que, ainda existe uma faixa de vegetação circundando toda a vereda e vazios urbanos com vegetação rasteira.

A Vr3 possui algumas características que também a distingue estatisticamente das demais áreas, como a similaridade na distribuição dos pontos amostrados. Esse resultado corrobora com o que pode ser observado em campo. Esta área alagada está inserida em uma Reserva Ecológica dentro de uma área de Reserva Legal, com vegetação natural preservada, poucos sinais de intervenção antrópica e visualmente diferente das demais áreas monitoradas.

\section{CONCLUSÕES}

Neste diagnóstico foram utilizados métodos estatísticos multivariados para avaliação da qualidade dos sedimentos em veredas da bacia do rio Uberabinha. A PCA do conjunto de elementos metálicos reduziu a matriz inicial de dados de 18x34 para 18x4, ou seja, foram extraídas quatro PC, que permitiram identificar a associação dos elementos, além da diferenciação entre as áreas alagadas monitoradas.

As análises dos metais nos sedimentos mostraram que as áreas foram associadas em três grupos, sendo um formado pela $\mathrm{Vr}$, outro pela Vu1 e o terceiro por uma associação dos pontos das demais veredas. Com isso, pode-se dizer que as atividades antrópicas no entorno da Vu2 ainda não são intensas a ponto de modificar significativamente suas características; que as principais características dos ecossistemas de vereda estão sendo alteradas na Vu1 em decorrência de atividades antrópicas; e que as condições de preservação da vegetação na $\mathrm{Vr} 3$ a difere estatisticamente das demais áreas, assim como pode ser observado in situ.

Apesar do estudo ter demonstrado a utilidade dos métodos estatísticos multivariados na avaliação da qualidade dos sedimentos, essas análises por si só não são suficientes. O conhecimento da geologia local e dos usos e ocupações do solo na bacia, são informações importantes no diagnóstico de impactos. Ressaltase a importância de estudos mais aprofundados assim como este, pois somente com o entendimento do papel ambiental desses ecossistemas e a disseminação do conhecimento é que será possível adotar políticas adequadas e mais assertivas.

\section{REFERÊNCIAS}

ALVARES, C. A.; STAPE, J. L.; SENTELHAS, P. C.; GONÇALVES,

J. L. M.; SPAROVEK, G.. Köppen's climate classification map for Brazil. Meteorologische Zeitschrift, v.22, n.6, p.711-728, 2013. 
ARAÚJO, I. M. M.; OLIVEIRA, Â. G. R. C.. Agronegócio e agrotóxicos: impactos à saúde dos trabalhadores agrícolas no nordeste brasileiro. Trabalho, Educação e Saúde, v.15, n.1, p.117-129, 2017. DOI: http://doi.org/10.1590/1981$\underline{7746-50 l 00043}$

BACCARO, C. A. D.. Unidades geomorfológicas do triângulo mineiro: estudo preliminar. Sociedade $\&$ Natureza, Uberlândia, n.3, p.5, 1991.

BACCARO, C. A. D.; FERREIRA, I. L. ROCHA, M. R.; RODRIGUES, S. C.. Mapa geomorfológico do Triângulo Mineiro: uma abordagem morfoestruturalescultural. Sociedade \& Natureza, Uberlândia, v.13, n.25, 2001.

CAIRES, S. M.. Determinação dos teores naturais de metais pesados em solos do Estado de Minas Gerais como subsídio ao estabelecimento de Valores de Referência de Qualidade. Tese (Doutorado em Solos e Nutrição de Plantas) Universidade Federal de Viçosa, Viçosa, 2009.

CAMPOS, M. L.; GUILHERME, L. R. G.; MARQUES, J. J. G. S. M.; CURI, N.; ARAÚJO, A. S. A.; MIQUELLUTI, D. J.; LOPES, C.; SPIAZZI, F. R.. Teores de arsênio e cádmio em solos do bioma Cerrado. Revista Brasileira de Ciência do Solo, v.37, n.1, p.281-286, 2013. DOI: http://doi.org/10.1590/S0100$\underline{06832013000100029}$

CARVALHO, L. M. T.; SCOLFORO, J. R.. Monitoramento da Flora Nativa. In: WEIMAR, A. J. F.; CARVALHO, L. M. T.; MELLO, J. M.; OLIVEIRA FILHO, A. T.; OLIVEIRA, A. D.; SCOLFORO, J. R.; SILVA, C. P. C.. Inventário Florestal de Minas Gerais. Lavras: UFLA, 2008.

COSTA, F. P. M.; DUARTE, W. O.; NISHIYAMA, L.. Mapa das Permeabilidades do Solo da Bacia do Rio Uberabinha Elaborado a partir de Ensaios In: SIMPÓSIO BRASILEIRO DE CARTOGRAFIA GEOTÉCNICA E GEOAMBIENTAL, 6. Anais. Uberlândia: 2007.

CPRM. Serviço Geológico do Brasil. Mapa Temático Geodiversidade Minas Gerais. Belo Horizonte: CPRM, 2010.

FADIGAS, F. S.; AMARAL SOBRINHO, N. M. B.; MAZUR, N.; ANJOS, L. H. C.; FREIXO, A. A.. Concentrações naturais de metais pesados em algumas classes de solos brasileiros. Bragantia, v. 6, n. 2, p. 151-159, 2002. DOI: http://doi.org/10.1590/S0006-87052002000200008

FARRÉ, M.; KANTIANI, L.; PETROVIC, M.; PÉREZ, S.; BARCELÓ, D.. Achievements and future trends in the analysis of emerging organic contaminants in environmental samples by mass spectrometry and bioanalytical techniques. Journal Chromatography A, v.1259, n.12, p.86-99, 2012. DOI: https://doi.org/10.1016/i.chroma.2012.07.024

FÁVERO, L. P.; BELFIORE, P.. Manual de análise de dados: estatística e modelagem multivariada com Excel $^{\circ}$, SPSS $^{\circ}$ e Stata ${ }^{\circ}$. São Paulo: Elsevier Brasil, 2017.

FLAUZINO, F. S.. Qualidade da água e dos sedimentos nos reservatórios das Usinas Hidrelétricas de Nova Ponte e Miranda-Minas Gerais. Tese (Doutorado em Ciências Humanas) - Universidade Federal de Uberlândia, Uberlândia, 2014.
FREITAS, E. V. S.; NASCIMENTO, C. W. A.; GOULART, D. F.; SILVA, J. P. S.. Disponibilidade de cádmio e chumbo para milho em solo adubado com fertilizantes fosfatados. Revista Brasileira de Ciência do Solo, v.33, n.3, p.1899-1907, 2009. DOI: http://doi.org/10.1590/S0100-06832009000600039

GROSSI, S. R.. De Uberabinha a Uberlândia: os caminhos da natureza: Contribuição ao estudo da geomorfologia urbana. São Paulo: USP, 1991.

GUILHERME, L. R. G.; MARQUES, J. J.; PIERANGELI, M. A. P.; ZULIANI, D. Q.; CAMPOS, M. L.; MARCHI, G.. Elementos-traço em solos e sistemas aquáticos. In: TORRADO-VIDAL, P.; ALLEONI, L. R. F.; COOPER, M.; SILVA, A. P.. Tópicos em ciência do solo. Viçosa: SBCS, 2005. p.345-390.

GUIMARÃES, A. J. M.. Características do solo e da comunidade vegetal em área natural e antropizada de uma vereda na região de Uberlândia, MG. Dissertação (Mestrado em Ecologia e Conservação dos Recursos Naturais do Solo) Universidade Federal de Uberlândia, Uberlândia, 2001.

JACOB, D. L.; YELLICK, A. H.; KISSOON, L. T. T.; ASGARY, A.; WIJEYARATNE, D. N.; SAINI-EIDUKAT, B.; OTTE, M. L..

Cadmium and associated metals in soils and sediments of wetlands across the Northern Plains, USA. Environmental Pollution, v.178, n.1, p.211-219, 2013. DOI: http://doi.org/10.1016/i.envpol.2013.03.005

MARQUES, J. J.; SCHULZE, D. G.; CURI, N.; MERTZMAN, S. A.. Trace element geochemistry in Brazilian Cerrado soils. Geoderma. v.121, n.2, p.31-43, 2004. DOI: http://doi.org/10.1016/j.geoderma.2003.10.003

MCGRATH, S. P.; CUNLIFFE, C. H.. A simplified method for the extraction of the metals $\mathrm{Fe}, \mathrm{Zn}, \mathrm{Cu}, \mathrm{Ni}, \mathrm{Cd}, \mathrm{Pb}, \mathrm{Cr}, \mathrm{Co}$ and $\mathrm{Mn}$ from soils and sewage sludge. Journal of the Science of Food and Agriculture, v.36, n.9, p.794-798, 1985. DOI: https://doi.org/10.1002/jsfa.2740360906

MEIRELLES, M. L.; GUIMARÃES, A. J. M.; OLIVEIRA, R. C.; ARAÚJO, G. M.; RIBEIRO, J. F.. Impactos sobre o estrato herbáceo de áreas úmidas do Cerrado. In: AGUIAR, L. M. S.; CAMARGO, A. J. A.. Cerrado: ecologia e caracterização. Planaltina: EMBRAPA, 2004. p.41-68.

MENDES, A. M. S.; DUDA, G. P.; NASCIMENTO, C. W. A.; SILVA, M. O.. Bioavailability of cadmium and lead in a soil amended with phosphorus fertilizers. Scientia Agricola, v.63, n.2, p.328-332, 2006. DOI: http://doi.org/10.1590/S010390162006000400003

MINAS GERAIS. Deliberação Normativa COPAM n.166 de 29 de junho de 2011. Belo Horizonte: DOE, 2011.

MINAS GERAIS. Lei $\mathbf{n . 2 0 9 2 2 ~ d e ~} \mathbf{1 6}$ de outubro de 2013

Dispõe sobre as políticas florestal e de proteção à biodiversidade no Estado. Belo Horizonte: DOE, 2013.

MUEHE, E. M.; ADAKTYLOU, I. J.; OBST, M.; ZEITVOGEL, F.; BEHRENS, S.; PLANER-FRIEDRICH, B.; KRAEMER, U.; KAPPLER A.. Organic carbon and reducing conditions lead to cadmium immobilization by secondary Fe mineral formation in a $\mathrm{pH}$ neutral soil. Environmental Science \& Technology, v.47, n.23, p.13430-13439, 2013. DOI: https://doi.org/10.1021/es403438n 
OTTO, M.. Chemometrics: statistics and computer application in analytical chemistry. Hoboken: John Wiley \& Sons, 2016.

PAYE, H.; MELLO, J. W. V.; ABRAHÃO, W. A. P.; FERNANDES FILHO, E. I.; DIAS, L. C. P.; CASTRO, M. L. O.; MELO, S. B.; FRANÇA, M. M.. Valores de referência de qualidade para metais pesados em solos no Estado do Espírito Santo. Revista Brasileira de Ciência do Solo, v.34, n.6, 2010. DOI: http://doi.org/10.1590/S0100-06832010000600028

RAMOS, M. V. V.; CURI, N.; MOTTA, P. E. F.; VITORINO, A. C. T.; FERREIRA, M. M.; SILVA, M. L. N.. Veredas do Triângulo Mineiro: solos, água e uso. Ciência e Agrotecnologia. v.30, n.2, p.283-293, 2006. DOI: http://doi.org/10.1590/S141370542006000200014

RAMOS, M. V. V.; HARIDASAN, M.; ARAÚJO, G. M.. Caracterização dos Solos e da Estrutura Fitossociológica da Vegetação de Veredas da Chapada no Triângulo Mineiro. Fronteiras: Journal of Social, Technological and Environmental Science, v.3, n.2, p.180-210, 2014. DOI: http://doi.org/10.21664/2238-8869.2014v3i2.p180-210

REATTO, A.; CORREIA, J. R.; SPERA, S. T.. Solos do bioma Cerrado: aspectos pedológicos. In: SANO, S. M.; SEMÍRAMIS, P.; RIBEIRO, J. F.. Cerrado ecologia e flora. Brasília: EMBRAPA, 2008. p.107-149.

RIBEIRO, B. T.; NASCIMENTO, D. C.; CURI, N.; GUILHERME, L. R. G.; COSTA, E. T. S.; LOPES, G.; CARNEIRO, J. P.. Assessment of trace elements contents in soils and water from Cerrado wetlands, Triângulo Mineiro region. Revista Brasileira de
Ciência do Solo, v.43, n.1, 2019. DOI:

http://doi.org/10.1590/18069657rbcs20180059

RIBEIRO, J. F.; WALTER, B. M. T.. As principais fitofisionomias do bioma cerrado. In: SANO, M. S.; ALMEIDA, S. P.; RIBEIRO, J. F.. Cerrado: ecologia e flora. Brasília: EMBRAPA, 2008. p.151-199.

ROSOLEN, V.; CAMPOS, A. B.; GOVONE, J. S.; ROCHA, C.. Contamination of wetland soils and floodplain sediments from agricultural activities in the Cerrado Biome (State of Minas Gerais, Brazil). Catena, v.128, n.1, p.203-210, 2015. DOI: http://doi.org/10.1016/j.catena.2015.02.007

SHUQAIR, M. S. S.. Estudo da contaminação do solo e água subterrânea por elementos tóxicos originados dos rejeitos das minas de carvão de figueira no Estado do Paraná. Tese (Doutorado em Ciências na Área de Tecnologia Nuclear) Universidade de São Paulo, São Paulo, 2002.

SILVA, L. S.; GALINDO, I. C. L.; NASCIMENTO C. W.A.; GOMES, R. P.; CAMPOS M. C.C.; FREITAS, L.; OLIVEIRA, I. A.. Heavy metal contents in Latosols cultivated with vegetable crops. Pesquisa Agropecuária Tropical, v.46, p.391-400, 2016. DOI: http://doi.org/10.1590/1983-40632016v4641587

USEPA. United States Environmental Protection Agency. Method 3051A: microware assisted acid digestion of sediments, sludges, soils and oils. Washington: USEPA, 1998.

USEPA. United States Environmental Protection Agency. Soil screening guidance: Technical background document. Washington: USEPA, 1996.

A CBPC - Companhia Brasileira de Produção Científica (CNPJ: 11.221.422/0001-03) detém os direitos materiais desta publicação. Os direitos referem-se à publicação do trabalho em qualquer parte do mundo, incluindo os direitos às renovações, expansões e disseminações da contribuição, bem como outros direitos subsidiários. Todos os trabalhos publicados eletronicamente poderão posteriormente ser publicados em coletâneas impressas sob coordenação da Sustenere Publishing, da Companhia Brasileira de Produção Científica e seus parceiros autorizados. Os (as) autores (as) preservam os direitos autorais, mas não têm permissão para a publicação da contribuição em outro meio, impresso ou digital, em português ou em tradução. 